\title{
14
}

\section{Inside and Out: Violence against Women and Spatiality in Timor-Leste}

\section{Damian Grenfell ${ }^{1}$}

\section{Introduction}

Violence in Timor-Leste has been a continuous point of analytical inquiry, not least given the consequences of Portuguese colonialism, Indonesian occupation and the civil strife that ebbed and flowed in the new republic from 2006 to 2008. Since independence, efforts to reduce violence against women have been constant, even if much of this has been in the form of advocacy competing with other post-independence agendas. Despite innumerable programs and associated research, all available evidence suggests that violence against women is not abating. According to the Timor-Leste Demographic and Health Survey published in 2010, 38 per cent of women in Timor-Leste aged 15 to 49 have experienced physical violence. ${ }^{2}$ The 2016 study Understanding Violence against Women and Children in Timor-Leste found that 59 per cent of ever-married women aged 15 to 49 had experienced physical and/or

\footnotetext{
1 My appreciation to colleagues both in Timor-Leste and Australia, not least those who contributed to research for the 'Beyond Fragility and Inequity, The Economic Dimensions of Domestic Violence in Timor-Leste' project, which provided several ideas further developed here.

2 National Statistics Directorate, Timor-Leste Demographic and Health Survey, 229.
} 
sexual violence by a male intimate partner at least once, though a large majority (81 per cent) had experienced intimate partner violence many times. ${ }^{3}$ Entrenched patriarchies and gender inequity remain central to this problem, and responding to these in the context of what is referred to here as a 'hybrid order' adds variously to the challenges of addressing this violence.

The key argument of this chapter is that violence against women and the interventions to prevent it shape and are shaped by different forms of space within a hybrid order. This is just one of the possible conceptual deployments of hybridity in a site such as Timor-Leste where customary social relations have continued to play a significant role in patterns of social integration and political life. ${ }^{4}$ As a concept, hybridity creates space for analysis of different forms of sociality that do not clearly sit within the domain of what may be described as 'modern'. Rather than seeing the world through the narrow and flat lens of modernity-for instance, via state-centric approaches-it draws analysis towards an examination of constituent elements that in turn contribute to understanding the character and complexity of everyday sociality.

The first two sections of this chapter provide an initial discussion of hybridity, setting out the constituent elements focused on here as the modern and the customary, and in turn discussing how these relate to spatiality. Building on this, the third section argues there is a tendency for exogenous forces - such as donors, non-government organisations, aid agencies and academics - to 'render' East Timorese society as patriarchal, establishing a point of difference that in turn justifies the shape of interventions. The fourth and fifth sections shift the focus to the control of space where women are subjected to a kind of 'double sovereignty'. In the context of Timor-Leste, the 'double' refers to the fact that women are constrained across both modern and customary spatialities, with sovereignty used to denote the control over space, in this instance masculine control expressed through patriarchal power that effectively limits the mobility of women. This approach to sovereignty is not to be confused with liberal notions of popular or individual sovereignty but, rather, is an adaptation of a Weberian notion of control over a particular territorial formation and the relationship to violence. While this form of sovereignty is often discussed as part of debates on globalisation where it is understood

3 Asia Foundation, Understanding Violence against Women and Children in Timor-Leste.

4 Wallis, 'A Liberal-Local Hybrid Peace Project in Action?'. 
as 'the extension and institutionalization of control and authority within a spatial field', here sovereignty is used to describe the control of space within 'petit polities' that exist quite apart from the nation-state system. ${ }^{5}$ As power is concentrated in masculine identities, sovereign control is expressed via men over different kinds of space, limiting pathways for help and support for women who are experiencing abuse. Underpinning each of the arguments in this chapter is a concern that modernity, especially in the context of a hybrid order, does not necessarily provide a pathway for women to reduce the risk and experience of abuse.

\section{Hybridity and Timor-Leste}

Not without some irony the concept of 'hybridity' has largely shed its association with its racist heritage. ${ }^{6}$ Having in recent decades become more prominent in the social sciences and humanities, hybridity allows for a greater identification of sources of power and identity that are distinct from modern political and social domains. It has largely gained traction as a way to counter depictions of 'state-failure' and of 'ungovernable' populations, particularly in postcolonial states that have experienced large-scale violence including contexts where the modern state is unevenly constituted.

Hybridity in this chapter is taken as a way of explaining contexts where multiple patterns of social integration-or 'life-worlds' as they will be referred to in shorthand-are in evidence to the extent that it is difficult to claim that one has a clear dominance over another. Instead, it is taken that there are multiple forms of sociality of significance; 'lifeworlds' that speak to the embedded assumptions that shape patterns of practice. Cohen and Arato describe a 'life-world' as 'the reservoir of implicitly known traditions, the background of assumptions that are embedded in language and culture and drawn upon by individuals in everyday life'. ${ }^{8}$ This chapter follows in similar sense, with the emphasis on the relationships between people (social integration) as manifest in the patterns of everyday practice. Extending further than the domains

\footnotetext{
5 Agnew, Globalization and Sovereignty, 2.

6 Dinnen and Kent, 'Hybridity in Peacebuilding and Development'.

7 Boege et al., 'Hybrid Political Orders, Not Fragile States'; Mac Ginty, International Peacebuilding and Local Resistance; Mac Ginty and Richmond, 'The Fallacy of Constructing Hybrid Political Orders'; Mallet, 'Beyond Failed States'; Richmond, 'Post-colonial Hybridity'.

8 Cohen and Arato, Civil Society and Political Theory, 427.
} 
of language and culture, however, these include socioeconomic and sociopolitical practices, and in turn encompass what these 'assumptions' are across space, time and epistemology.

A hybrid order refers to contexts where differing life-worlds are each copresent to significant extents, with the focus here on the 'customary' and 'modern'. The customary reaches beyond culture, and in Timor-Leste is a way of describing a world view that reverberates through forms of exchange, production, organisation, communication and so on, framed by a cosmological view of the world bound very strongly to a sense of origin. The modern, however, is grounded in different forms of practice (for instance, the dominance of mass digital communication over the oral, technocratic leadership over genealogical authority), and where secular logic and rationality are the dominant ways in which society is integrated and ordered. This pushes the analytical lens past ideology for instance, which is itself a manifestation of modernity, and thus debates about the liberal peace are understood here as one possible manifestation of a modernity in which the individual and a particular form of state (largely Weberian) come to the fore. ${ }^{9}$

It is difficult to claim that either the customary or the modern has a particular dominance in Timor-Leste. While speaking of 'Timor-Leste' is an immediate reference to a modern political formation, other ways of organising social life remain vitally important across the territory. The customary — often cast discursively as traditional, indigenous, local or even 'culture' - on one hand, and the modern on the other, give an account of important moments in the texture and contours of social life. These terms are nevertheless heuristic devices used here to allow for analysis that distinguishes an ontological basis for patterns of practice. Analysis is an abstracting process and the separation of social life into categories (these, or others such as the state and civil society, or the local and the global) does not tend to exist in the same way in terms of how people see their own actions in an everyday sense. As such, these categories allow for analysis to occur, but are not necessarily representative of the ways people would readily see themselves or categorise their practice in the first instance (which can begin to occur when people are asked questions by researchers). In turn such categories do not necessarily create binaries, though analysis that assumes, for instance, there is a tension between

9 Steger, The Rise of the Global Imaginary, Chapters 1-3. 
the two tends in that direction, and similarly where analysis implicitly or explicitly claims there are only two social possibilities: the modern and the otherwise (however categorised). As such, while drawing to these two categories of social integration, they do not present an overall unity nor claim to explain all the different dimensions of sociality. These are merely two points of emphasis that are relevant in Timor-Leste.

\section{Customary and modern spatiality}

The endogenously and exogenously driven modernising efforts that occur in Timor-Leste have not resulted in a demise of customary social relations. ${ }^{10}$ The customary and modern are often seen to create points of friction, or entanglement, in forms of governance, systems of exchange and - as per the following quote from a 2003 report—at the intersection of justice processes and gender relations:

Women leaders feel that the use of local justice systems is 'not better that [sic than] nothing' as it undermines attempts to implement standards of law which might combat factors preventing women from accessing it, such as powerlessness and shame. However, inherent cultural practices, beliefs and norms are so powerful that even with education in the era since Independence, standards of human rights and other modern concepts are being both misunderstood and completely lost due to the massive gap between these concepts and the realities on the ground. ${ }^{11}$

While the quote suggests tension between two life-worlds, it also gives rise to a sense of one being drawn into and reframed by the other (in this instance as modern concepts are 'misunderstood'). More than a decade after this was written, women continue to navigate narrow possibilities across two forms of justice to the extent that some have called for a formalised negotiation between different legal systems. ${ }^{12}$ Rather than examine the process of justice itself, this chapter takes a different route and argues that each 'system' is situated in a life-world and in analytical terms underpinned by different forms of epistemology, temporality and, as the focus here, spatiality.

10 Carroll-Bell, 'Development Alternatives in Timor-Leste'; Hohe, 'The Clash of Paradigms'; McWilliam, 'Houses of Resistance in East Timor'.

11 Swaine, Traditional Justice and Gender Based Violence, 14.

12 Kovar and Harrington, Breaking the Cycle of Domestic Violence in Timor-Leste. 
Space is an abstraction that enables humans to order their relationship to both the material world and to one another. Space is distinguished by bounded areas that mark that which is inside and outside, and can be materially defined — for instance, the marked boundaries to land- to that which is seemingly immaterial: an online community, a network, a national space, a public sphere. As the existence of boundaries suggests, there are always multiple spaces; these layers cut across one another and, following Massey, are in a constant state of reproduction:

First, that we recognise space as the product of interrelations; as constituted through interactions, from the immensity of the global to the intimately tiny ... Second, that we understand space as the sphere of the possibility of the existence of multiplicity in the sense of contemporaneous plurality; as the sphere in which distinct trajectories coexist; as the sphere therefore of coexisting heterogeneity ... Third, that we recognise space as always under construction. Precisely because space on this reading is a product of relations-between, relations which are necessarily embedded material practices which have to be carried out, it is always in the process of being made. It is never finished; never closed. ${ }^{13}$

In turn, 'spatiality' is taken here as referring to the relationship between social life and space. It is more than the domain itself in a dimensional sense, referring to the social relations that constitute that space. Its use here is similar to Silva's approach to place in her work on Dili and the 'mountains' in Timor-Leste, where she uses 'the category place as a morally meaningful space to which certain actors and agencies are associated'. ${ }^{14}$ While place here is understood as a fixed and identified geographic location, spatiality takes on the moral dimensions that Silva speaks to as well as other elements of social life, such as knowledge, rights and history.

One way of thinking about a hybrid order is conceiving it as a multitude of spatialities composed both across and within different patterns of practice that are in a constant state of reproduction and change. Here, then, to speak of customary and modern forms of spatialities is to draw back to a metatype that allows for generalised analysis, though as any inquiry becomes more specific the more immediate context would shape and contour any description, adding nuance and variation.

13 Massey, For Space, 9.

14 Silva, 'Foho versus Dili', 146. 
Customary space in an East Timorese context could be characterised by interrelationships in two ways: the genealogical and kinship connections between living people, and in turn their relationship with the ancestral domain. Space and place-the latter being the specific identified geographic location - tend to be closely proximate, their boundaries may not be contiguous, they tend to incorporate sacred sites that are exclusive to a specific group, and they are often associated with a powerful sense of origin. The ground, or land in a more generalised sense, tends to be of intense importance in this form of space.

Modern space, in the alternative, is treated as secular, empty, commodifiable, transferrable, unifiable and homogenous, and tends to be sharply delineated. To return to 'Timor-Leste', 'territory' is a form of modern space where what might have been fairly open frontiers are now hard borders measured to the centimetre by satellite systems.

In the remainder of this chapter I argue that in the context of a hybrid order different spatialities inform the patterns of violence against women. Challenges lie with external agencies and how they approach gender more broadly in Timor-Leste and, as discussed in the next section, different forms of spatiality may be transgressed in ways that could undermine the broader objectives of ending violence against women.

\section{Rendering Timor-Leste}

A recently published article opened with the explanation that 'patriarchal traditions and a history of armed conflict in Timor-Leste provide a context that facilitates violence against women. ${ }^{15}$ The sentence is ambiguous enough to avoid the claim of direct causality, though the reader is left very much with the impression that violence against women is inextricably linked to two localised characteristics, namely 'tradition' and 'history'. Such a claim is hardly exceptional as Timor-Leste is frequently described as a 'patriarchal society', referring to the fact there is a clear power inequity between women and men that transpires in a myriad of ways. While the participation of women is regulated on local governing councils

15 Meiksin et al., 'Domestic Violence, Marital Control, and Family Planning, Maternal, and Birth Outcomes in Timor-Leste', 1338. 
(konsellu suku), and Timor-Leste leads the way in terms of participation of women in parliament, this has not translated into shifting power imbalances more generally. ${ }^{16}$

The key concern here is that while it is possible to classify Timor-Leste as patriarchal, when it is done as a broad descriptor-and as an implicit differentiator from other societies-then the effect is a powerful one. If it is accepted that virtually all societies reflect significant patterns of patriarchy, then differentiating on these grounds appears, when undertaken by outsiders, to establish points of separation rather than creating opportunities that allow for meaningful connections to be made. This is a kind of 'rendering' that labels a population negatively and as a consequence approaches it as if it is in need of treatment. ${ }^{17}$ To turn this around and speak of 'patriarchy in an East Timorese context' may appear only to make a minor discursive alteration, though here it is argued otherwise.

In the first instance, assertions of Timor-Leste as patriarchal per se and as an all-encompassing categorisation risk missing the agency of those who struggle against it, and in turn the identification of subsequent counterspaces. Such an approach also closes rather than opens pathways to build spaces for solidarities and mutual learning across societies. By contrast, rather than concentrating only on points of difference, acknowledging similarities may promote esteem and disable shame or self-blame if survivors of violence come to understand that they are not an exception, either individually or as a defined group.

Second, rendering Timor-Leste as patriarchal provides a kind of legitimating logic for a particular form of intervention into spaces that would otherwise be largely inaccessible to outsiders. Naming patriarchy_or the less confronting surrogate 'gender inequity' - as a societal designator provides the raison d'être for foreign-funded programs to justify interventions. One of the challenges of working on violence against women is that it confronts entrenched forms of power and control in very embodied ways that call an intimate sense of self into question: sex and sexuality, fertility and procreation, household economies, spirituality and faith, the regulation of the body. These are not necessarily based in a customary 'life-world', but in Timor-Leste they often are. Establishing points of

16 Cummins, 'The Problem of Gender Quotas'.

17 Pupavac, 'Therapeutic Governance'. 
difference then equips the outsider with a rationale for challenging the very body and how it is understood, not least as they become focused at the same points of intimate life; into health and reproduction, or gender norms and behaviours, relationships and so on. These may or may not be helpful in and of themselves, but the point here is the danger in the assumed legitimacy of doing so and the onward effects.

To come back to the hybrid order discussed above, such a form of intervention in Timor-Leste can be seen as occurring across two forms of spatiality. In one way, the modernity of interveners-non-government organisations, aid and humanitarian workers, state agencies-often correlates to the way that practice is matched to conceptions of space. That is, programs are planned to forge social change within a modern space. As a result, activities are directed at the public sphere, into a civil society, through the state, into localised public spaces, school curriculums and so on. Despite the limited reach of television in Timor-Leste, TV commercials are made depicting 'Feto Fantastiku', a superhero woman dressed in a cape made of local weaving. Banners are hung across busy streets, posters placed at the offices of local leaders, T-shirts printed. Existing norms are contested as spatiality is imagined as the modernist either sees it or wills it to be: open, accessible, contestable via evidence and arguments of universal rights, and with the potential of carrying an authoritative voice via disembodied means.

Modern spatiality has remained the preoccupation of foreign-led interventions, though the justification of patriarchy also allows for a transgression of customary spatiality. By this it is meant there is a sense of a legitimate claim to penetrate social relations where access would otherwise be unlikely, such as challenging the role of customary leaders or social priorities (such as spiritual over material). This may be the case as 'culture' is seen as something that must change or simply because there is expatriate blindness to the existence of such a life-world at all. This is, in many respects, where the intervention is at its most powerful and helps us understand why discussions of violence in Timor-Leste often orient towards 'culture' and 'tradition'. The exchange process at the time of marriage, commonly termed barlake in Tetun, is an example of a discursive signifier that carries a sense of oppression in spite of counterevidence. With the origins of violence against women then localised (doubly so when history of warfare is included), the conditions are set in 
place where demands for change are focused inwards and across space that would otherwise be untraversable by the foreigner. The article quoted at the opening of this section ends up calling for exactly this outcome:

Our findings suggest that in the short-term, targeted interventions addressing family planning, maternal healthcare, and birth outcome vulnerabilities for Timorese women who have experienced DV or marital control would be a good use of public health resources, due to their elevated risk. ${ }^{18}$

There is a need here to be unequivocal about what is being argued. It is entirely appropriate that there are aid-funded programs that work to support survivors of domestic violence, both specifically in terms of support services and prevention, as well as in health, justice, policing and so on. The challenge then is that this needs to be positioned as emancipatory rather than as a form of societal control, and this is far more difficult to achieve if the broad logic is shaped by differentiation rather than mutuality.

Over the decade or more since independence, it is possible to see, for instance, that resistance to the idea of gender, and gender equity, has developed within local populations and targeted as an 'outside' or foreign idea. ${ }^{19}$ Contestation is to be expected, and in some instances suggests a more rigid application of culture as men attempt to maintain control. ${ }^{20}$ However, that 'gender' is often deemed a foreign concept that came after independence and is contrary to 'local culture' is also in part a dynamic generated by the way programs have been framed. ${ }^{21}$ Carrying a thread of the colonial encounter, power rests on maintaining differentiation and the demands for change cut into spheres of life without generating the inside legitimacy that enable traction. Simultaneously this dynamic demands virtually no reflexivity on the part of the intervener, for instance, in terms of exploring the reasons why violence against women remains so deeply intractable in societies typically designated as 'modern'. In such circumstances, differentiation in this form will more likely lead either to internalisation or resistance, neither productive in terms of ending violence against women and providing support to those who experience it.

18 Meiksin et al., 'Domestic Violence, Marital Control, and Family Planning', 1346.

19 UNFPA, Gender-Based Violence in Timor-Leste.

20 Niner, 'Hakat Klot, Narrow Steps'.

21 Smith, 'When “Gender” Started', 56. 


\section{Customary spatiality and double sovereignty}

A recently published Asia Foundation report found that women were often contained to a 'domestic sphere' where, for a combination of economic and political reasons, it was difficult for them to leave or seek assistance when experiencing abuse. ${ }^{22}$ Extending this analysis, the argument across the last two sections of this chapter is that while economic factors are central, they are one part in a pattern of control that is referred to as 'double sovereignty', a term used to explain how a woman's mobility is contained and controlled across both customary and modern spaces. Sovereignty, as discussed in the Introduction, denotes a control over space, and while typically applied in the context of nation-states, here it refers to localised forms of spatiality that set the perimeters for the possibilities of everyday life. ${ }^{23}$

To turn to a discussion of the first element of this double sovereignty, a key aspect of customary spatiality in Timor-Leste is that access to space is based on genealogical and kinship ties. Legitimacy to participate in social life is dependent on how one claims a place in relation to extended familial networks and in turn access to space that is connected to production (the home, land, water, forests, people), spirituality (land, lulik sites, graves, ancestors), knowledge (elders, sacred houses), exchange of commodities (extended family, including ritual belongings) and communication (people, embodied communal space).

Forms of customary spatiality are reproduced in a myriad of localised ways, both through designated sacred sites and at moments of ritual, as well as in terms of cosmological understandings of land and ancestors and conceptions of agency, power and control. As such it is also manifest in an unannounced way in how patterns of social life are constantly re-created. These come to the fore at the point of intersection between familial groupings (uma kains, as extended clans defined by genealogical and kinship connection). For instance, people announce their sacred house as they arrive at funerals, name sacred houses to denote precedence in relation to land, and terms such as kaben tama (the male enters the wife's family on marriage) and kaben sai (the female leaves her family at marriage) are used to map the boundaries of inclusion and exclusion of families.

22 Grenfell et al., Beyond Fragility and Inequity.

23 Agnew, Globalization and Sovereignty. 
This form of spatiality operates as a series of petit polities thatas discussed in the Introduction-are formed around a kind of spatial sovereignty. Outsiders rarely transgress these in any kind of permanent way (and even a temporary transgression would often require some kind of negotiation). In Timor-Leste, migration without existing connections is uncommon where customary spatialities remain dominant. Customary regulations (lisan or adat) are seen to regulate households in ways that separate one extended familial unit from another. 'According to our adat' is a common preface to sentences. Moreover, there is a constant reference to matters being kept 'within the household', generally meaning either an immediate family (familia rasik) or a more extended familial unit bound by genealogy and affinal relations, and ordered through association to sacred houses. Hence, familial units are bound into different forms of sovereignty that are exclusive from one another.

There is a range of ways that customary forms of spatiality can be seen as reproducing patriarchal social relations. These can be seen as most clearly manifest in the overwhelming dominance of males in leadership positions (across the spectrum of spiritual and political), divisions of labour, control over the body, norms around procreation and mobility. The counterexamples of matrilineal systems in Timor-Leste are both exceptional and shift the texture of power but not the power itself. ${ }^{24}$

In this context, sovereign control embedded in masculine identities, and manifest in the relationship between women and men, is exercised to control a women's mobility through economic dependence, divisions of labour, patterns of procreation and forms of coercion. However, the sovereignty also means a resistance to external intervention because 'outsiders'-whether other East Timorese or foreigners-have no legitimacy to reach into the particularities of customary space. The outside world has restricted access, and, when it does enter, it does so frequently as negotiated by males such as where domestic violence cases are referred out to police and then back to the families. The 2010 Law Against Domestic Violence is designed to interrupt this space by mandating that all reported cases are investigated by the police, effectively pulling the inside-customary into the outside-modern space of the nation. However, this only works to a certain extent, not just because of resources but also because the police, health workers and others at the interface of intervention straddle the customary and modern themselves.

24 Grenfell et al., Beyond Fragility and Inequity, 56-68. 


\section{Modern sovereignty and urban violence}

The unevenness between the co-existing forms of spatiality in Timor-Leste does not necessarily shift the possibilities for women who are experiencing violent abuse. Despite the concentrations of law enforcement, support agencies, communication systems and transportation, rates of violence against women remain high in urban centres such as Dili.

The results show that intimate partner violence is a significant problem in all sites across Timor-Leste. However, rates of physical and sexual intimate partner violence were consistently higher in Dili than in other municipality sites, and higher in urban areas compared with rural areas. For example, in Dili 64 percent of ever-partnered women had ever experienced physical and/or sexual intimate partner violence in their lifetimes, compared with 57 percent in other sites. ${ }^{25}$

In part to account for this, I argue here that modern forms of spatiality remain patriarchal and represent the second element or tier of a 'double sovereignty' that results in the control over women's mobility. Critical to this is the way a sharp distinction emerges between public and private spaces as different articulations of modern spatiality.

In a predominantly urban site such as the capital, Dili, a modern spatiality comes to the fore via public spaces that are no longer genealogically determined: roads and pathways, transport, institutional formations such as universities, the public service and hospitals, public parks and soccer fields, commercial enterprises and, more abstractly, a public sphere via digital and print media. The non-contiguous boundaries of community often found in rural areas change for the more rigid boundaries dividing urban suku from each other. ${ }^{26}$ At the same time, the notion of a private sphere becomes grounded in the intimate family, and family size tends to diminish as does the ability to co-locate extended families. 'Property rights' designate access to bounded land while emergent 'liberal rights' defend against an intrusive state.

A commonality between customary and modern spatialities is found via the way that women remain contained overwhelmingly to the domestic sphere, albeit for different reasons. The allure of cash income within modern modes of production is sustained by unpaid labour at home

25 Asia Foundation, Understanding Violence against Women and Children, 51-52.

26 Grenfell et al., Understanding Community. 
including care for children. Such activities are consistently mapped via essentialised notions of gender, and the private sphere becomes a new domain of containment. The result can be both a lack of access to the means of economic production (especially as women become disengaged from land) and a continued if not accelerated dependency on intimate partners, with an effect on gendered identity formations.

The significantly lower number of income-generating activities carried out by women in Dili, alongside the shift in perceptions that women's role is solely that of 'housewife' rather than 'farmer,' could suggest an increase in women's dependency as urbanisation increases. During participatory rural appraisals and focus group discussions in Dili, women tended to emphasise women's role as primary caregiver and were more likely to state that the woman's role was in the home whereas the man's role was to generate income. This type of statement was rarely heard in the more rural settings. ${ }^{27}$

In speaking of the containment of women's mobility, True points to the way some women in the global North offset their own risk of intimate partner violence by employing women from the global South:

In a mutually constitutive way, the strict division of roles in the domestic sphere constrains women's public participation and their access to economic opportunities in the market, in turn creating inequalities in household bargaining power between men and women and entrapping women into potentially violent environments at home and at work. Some women, especially those in developed countries, avoid patriarchal, and potentially violent, situations in the family/private sphere by contracting out care work to poor women, including migrant women from the global South. ${ }^{28}$

Even without crossing national borders, a similar dynamic can often be seen in sites such as Dili. In cases where women find work and increase their economic independence, the need for unpaid labour as a carer for the home and children remains with them. In such cases young women, very often at some point of familial connection, are moved from rural communities to Dili and work at very low rates (or even just for board and meals). Where a male is employed but unmarried, then a female from the extended family will often live with him in order to cook and clean. Either way, there is a constant reproduction of modern private spheres being gendered as the essential domain for women.

27 Grenfell et al., Beyond Fragility and Inequity, 54.

28 True, 'The Political Economy of Violence against Women', 45. 
One of the features of modernity is the hard distinction that tends to emerge between public and private. In addition to the private sphere then, the public sphere is a second form of modern spatiality where patriarchal norms are consolidated. In the independence period in Timor-Leste, militaristic masculinities have become vaunted and celebrated via the heroic warrior image associated with the republic's veteran leadership. ${ }^{29}$ The creation of ideal forms emerge as marketing becomes more important, as seen at the icon of high modernity, the 'Timor Plaza' shopping centre parades of young women in 'Little Miss Timor' competitions. Nation and gender are connected via abstracted norms of the ideal woman.

While there are always exceptions and degrees of variation, the argument is that men often maintain a kind of sovereign control over modern forms of spatiality. Women remain overwhelmingly contained to the domestic sphere, redoubled by the essentialising symbolic trajectories in the public sphere. Mobility for women may of course be possible, but it is regulated, granted permission and 'chaperoned'. Women might well, for instance, gain an education, but there will be immense pressure for children at some point regardless, and even in the event of professional work they will remain responsible for the home sphere. This creates an environment where women often remain financially dependent and, in periods of abuse, with restricted ability to leave. At the same time in this scenario it can be even more difficult to know where abuse is occurring, especially if the familial networks that can provide support within a customary polity have largely dissolved in the modern urban sphere.

\section{Concluding hybridity}

The effect of this form of 'double sovereignty' is profound in that it multiplies the range of inequities and challenges faced when violence against women occurs. Moreover, here two forms of spatiality have been discussed as separate categories that allow for an analysis as they delineate different ways people engage with space. For instance, a woman who migrates to an urban centre for work or marriage may be subject to new forms of containment and yet remain bound into customary socialities in her origin community. Albeit in different ways then, these forms

29 Kent, 'Remembering the Past, Shaping the Future'; Kent and Kinsella, 'A Luta Kontinua'; Niner, 'Hakat Klot, Narrow Steps'. 
of spatiality can overlap in order to compound the challenges faced, rather than provide pathways out of abuse, and be seen as sovereignty as control over women.

In summary then, I have drawn on the notion of hybridity-and more specifically a hybrid order-to consider how spatialities across different life-worlds exacerbate the experience of violence by women. The complexity of the hybrid order may allow for moments of navigation away from violence, though here the argument has been that-at least in terms of spatiality - neither modernity nor the customary necessarily provide a significant point of alleviation from violence. At a programming level, efforts to reduce levels of abuse that rest on designating populations as patriarchal and singularly localising, risk reducing longer-term efforts to prevent violence. These may allow for a form of justification for intervention, but may do so in a way that builds resistance as a kind of colonial encounter while failing to create a legitimate counterpoint to patriarchy locally. This is not to call for an end to interventions, rather it is an argument for them that is framed with a greater reflexivity on the part of outsiders with regard to their own modernity, and the role of violence and power within it. 
This text is taken from Hybridity on the Ground in Peacebuilding and Development: Critical Conversations, edited by Joanne Wallis, Lia Kent, Miranda Forsyth, Sinclair Dinnen and Srinjoy Bose, published 2018 by ANU Press, The Australian National University, Canberra, Australia.

doi.org/10.22459/HGPD.03.2018.14 